Colman, A. M., Pulford, B. D., \& Bolger, F. (in press). Asymmetric dominance and phantom decoy effects in games. Organizational Behavior and Human Decision Processes.

\author{
Asymmetric Dominance and Phantom Decoy Effects in Games \\ Andrew M. Colman ${ }^{a, *}$, and Briony D. Pulford ${ }^{a}$, Fergus Bolger ${ }^{b}$ \\ ${ }^{a}$ School of Psychology, University of Leicester, Leicester LE1 7RH, UK \\ ${ }^{\mathrm{b}}$ Durham Business School, Durham University, Mill Hill Lane, Durham DH1 3LB, UK
}

\begin{abstract}
In individual choices between alternatives $x$ and $y$, the availability of a third alternative $z$, judged inferior to $x$ but not to $y$, tends to increase preferences for $x$. Two experiments investigated corresponding strategic asymmetric dominance effects in games. In Experiment 1, 72 players chose strategies in six symmetric $3 \times 3$ games, each having one strategy dominating just one other, or in reduced $2 \times 2$ games constructed by deleting the dominated strategies. Asymmetrically dominated strategies, even when unavailable (phantom decoy), increased choices of the strategies that dominated them and bolstered decision confidence. In Experiment 2, 81 participants played 12 similar but asymmetric games with or without dominated strategies, and similar asymmetric dominance, phantom decoy, and confidence effects were found.
\end{abstract}

Keywords: asymmetric dominance, confidence, focal point, game theory, Property alpha, reason-based choice, strategic dominance

When choosing between alternatives $x$ and $y$, the availability of a third alternative $z$, to which the decision maker strictly prefers $x$ but not $y$, tends to increase the decision maker's preference for $x$ (Huber, Payne, \& Puto, 1982). For example, a shopper may feel indifferent between one cell phone $x$, costing $\$ 120$ with call charges of 15 c per minute, and another $y$, costing $\$ 100$ with call charges of $25 \mathrm{c}$ per minute, but the availability of a third option $z$, costing $\$ 140$ with call charges of 20c per minute, may cause the shopper to prefer $x$ to $y$, because $x$ is cheaper than $z$ on both relevant product attributes, whereas $y$ is not. This asymmetric dominance effect is large and robust, occurring not only under experimental conditions but also in real in-store purchases, and it has been reported even when the dominated alternative $z$ was presented as an unavailable phantom decoy option, like an item on a menu or catalogue that cannot be ordered because it is out of stock or unavailable (Ariely \& Wallsten, 1995; Doyle, O'Connor, Reynolds, \& Bottomley, 1999; Mishra, Umesh, \& Stem, 1993).

The implications of this phenomenon for interactive decisions in games have not previously been empirically investigated or apparently even discussed. The phenomenon of strategic dominance in games suggests a natural method of modeling a game-theoretic counterpart of asymmetric dominance. ${ }^{1}$ In a dyadic (two-player) game, one strategy dominates another for a particular player if it yields a higher payoff to that player irrespective of the strategy chosen by the co-player. Strategic asymmetric dominance is a feature of any game in which a player has one strategy that strictly dominates just one of two or more other strategies. A strategic asymmetric dominance effect may be said to occur if the presence of an asymmetrically dominated Strategy $z$ causes the player's preference for the dominating Strategy $x$ to increase relative to another Strategy $y$ in the game. A phantom decoy effect may be said to occur if an asymmetrically dominated Strategy $z$, shown but not available for choice, increases the player's preference for the dominating Strategy $x$. This article reports the results of two experiments 
designed to investigate whether strategic asymmetric dominance and phantom decoy effects occur in dyadic games with dominant strategies.

We examined dyadic games in which each player had three strategies, one of which strictly dominated just one other, and there were no other dominance relations between strategies. In one treatment condition, the asymmetrically dominated strategies were phantom decoys, visible but unavailable for choice, and in another they were available for choice, though inadmissible for rational (payoff-maximizing) players because they were strictly dominated, a generally accepted prediction of game theory being that if a game has a dominated strategy, then a rational player will not choose it. We compared strategy choices in these $3 \times 3$ games with choices in reduced 2 $\times 2$ versions of the games formed by deleting the dominated strategies.

The theoretical basis for our hypotheses, stated formally at the end of this introduction, is reason-based choice (Shafir, Simonson, \& Tversky, 1993; Shafir \& Tversky, 1992; Tversky \& Shafir, 1992). The key assumption is that, in decisions under uncertainty, when outcomes depend on unpredictable factors and there are no obvious rules to guide decisions, people tend to seize on any reason that may present itself for choosing one particular alternative rather than another. Our $2 \times 2$ control games afforded players no obvious reasons for choosing either strategy, and we assumed that players would therefore tend to lack confidence in their strategy choices. The expanded $3 \times 3$ versions, in contrast, each had one strategy strictly dominating one other, and this provided players with an obvious reason for choosing the dominant strategy. The reason for preferring a dominant strategy is that it is unconditionally better than the strategy that it dominates - irrespective of the co-player's choice - and this remains true even if the dominated strategy is a phantom decoy that is not available to be chosen, because an option that is unconditionally better than something else has at least something to recommend it. Property $\alpha$ and Savage's First Postulate

The asymmetric dominance effect seems obviously irrational, but it is not immediately obvious why this should be so. In Appendix A we show formally that it violates the basic contraction property of rational choice, Sen's (1969) Property alpha $(\alpha)$, and we prove that this property is implied by the first postulate of Savage's (1954/1972) theory of subjective expected utility (SEU), arguably the most fundamental axiom of rational choice. Informally, Property $\alpha$ asserts that if a decision maker considers a particular member of a set of alternatives to be the best, then that decision maker must also consider it to be best in any subset drawn from that set (for a critical discussion, see Sugden, 1985). Sen (1970) offered the following intuitive example of Property $\alpha$ : "If the world champion in some game is a Pakistani, then he must also be the champion in Pakistan" (p. 17). This example is flawed, because a chess player (for example) could win the world championship but lose the national championship of Pakistan. We suggest the following tidied-up example: If the oldest man in the world is in Pakistan, then he must also be the oldest man in Pakistan.

Property $\alpha$ is sometimes called "regularity" (Doyle, O’Connor, Reynolds, \& Bottomley, 1999; Huber, Payne, \& Puto, 1982; Luce, 1959; Tversky \& Simonson, 1993). A probabilistic version of it is assumed by Tversky's (1972) theory of elimination by aspects (see also Tversky \& Sattah, 1979) and by Luce's (1959) theory of individual choice. It is a special case of the independence of irrelevant alternatives (Chernoff, 1954; Fishburn, 1973; Nash, 1950). Luce and Raiffa (1957, p. 288) offered the following illustration of this more general principle. Suppose a customer in a restaurant, having studied a menu, considers ordering either salmon or steak and eventually decides on salmon. The waiter then informs the customer that fried snails are also available. The customer, who detests fried snails, responds to the waiter: "Splendid, I'll change my order to steak." This behavior seems manifestly irrational, because the addition of the fried snails option should be irrelevant to the customer's preference for salmon over steak. 
It turns out that any violation of Property $\alpha$ is also a violation of Savage's first postulate. This twofold postulate asserts, first, that for any pair of alternatives $x$ and $y$, a decision maker must consider either $x$ to be at least as good as $y$, or $y$ at least as good as $x$, or both, in other words the decision maker's preference relation must be complete; and second, that for any three alternatives $x, y$, and $z$, if a decision maker considers $x$ to be at least as good as $y$, and $y$ at least as good as $z$, then this decision maker must also consider $x$ to be at least as good as $z$, ensuring that the preference relation is transitive (Savage, 1954/1972, p. 18). See Appendix A for a formalization of this postulate and a mathematical proof that Property $\alpha$ is implied by it.

Most authorities consider Property $\alpha$ to be a fundamental characteristic of rational individual choice (see, e.g., Chernoff, 1954; Fishburn, 1973; Kreps, 1988; Luce \& Raiffa, 1957; Nash, 1950; Sen, 2002; Tversky \& Simonson, 1993), although it is not necessarily a characteristic of collective choice or of individual choice based on rationality criteria other than expected utility maximization (Sugden, 1985).

\section{Asymmetric Dominance in Games}

In strategic games, various contextual factors that might explain apparent violations of Property $\alpha$ are filtered out by the abstract representation of games. Because the payoffs represent utilities, any violation of Property $\alpha$ must necessarily represent a departure from the rationality assumptions of game theory.

Summarizing experimental evidence on individual decision making accumulated over two decades, Luce (1977) commented that "the only property of general choice probabilities that has not been empirically disconfirmed is regularity [Property $\alpha$ ]" (p. 229). The more recently discovered asymmetric dominance effect violates it, however, and the version of it that we have defined for strategic games may also violate it. A strategic asymmetric dominance effect may be shown to occur in games if players choose $y$ from the strategy set $\{x, y\}$ and $x$ from an expanded strategy set $\{x, y, z\}$ that includes an additional strategy $z$, strictly dominated by $x$ but not by $y$.

At an aggregate level, any significant increase in the relative frequency of choosing $x$ in the three-strategy case may be interpreted as evidence for a strategic asymmetric dominance effect. The frequencies in the two-strategy and three-strategy versions of the games can differ significantly from each other only if a significant proportion of players violate Property $\alpha$. A more powerful test could capitalize on the fact that the effect has been found to occur even when dominated alternatives are presented as unavailable phantom decoys that cannot be chosen (Doyle, O'Connor, Reynolds, \& Bottomley, 1999). There is persuasive evidence that irrelevant alternatives can influence decisions significantly (e.g., Bastardi \& Shafir, 1998; Carpenter, Glazer, \& Nakamoto, 1994; Shafir, Simonson, \& Tversky, 1993).

Preferences for dominant strategies have, of course, been extensively documented in experimental games. In particular, Cooper, DeJong, Forsythe, and Ross (1990) studied choices in several $3 \times 3$ games based on a fixed $2 \times 2$ game to each of which a third strategy, dominated by one or more of the original $2 \times 2$ strategies, was appended for each player. The embedded $2 \times 2$ game had two symmetric Nash equilibria, and the dominated third strategy varied from one $3 \times 3$ game to the next. The results showed that different dominated strategies, though they were seldom chosen, caused the players to prefer different Nash equilibria in the larger games. Cooper et al.'s study focused on factors affecting Nash equilibrium selection and not on asymmetric dominance or phantom decoy effects. Furthermore, it did not include a dominance-free control condition and therefore provided no evidence to show that the presence of a dominated strategy increases players' preferences for the strategy that dominates it, relative to the $2 \times 2$ game without the dominated strategy. 
According to the standard assumptions of game theory, rational (payoff-maximizing) players always avoid choosing dominated strategies and expect their co-players to avoid them also. On this basis, we formulated the following hypotheses. Hypothesis 1: The inclusion of asymmetrically dominated strategies will lead players to choose the strategies that dominate them significantly more frequently than in the $2 \times 2$ control condition. Hypothesis 2: The inclusion of phantom decoy dominated strategies that are visible but not available for choice will also lead players to choose the strategies that dominate them significantly more frequently than in the $2 \times 2$ control condition. In both cases, strategic dominance provides players with a reason for choice, although in the second case the reason rests on a strategically irrelevant context effect only. The experiments described below were designed to test both hypotheses. To facilitate the interpretation of the results, the experiments also examine the possible role of confidence in choices involving dominant strategies.

\section{Experiment 1}

Participants

The participants in Experiment 1 were 72 undergraduate students at the University of Leicester, most of them studying economics, mathematics, or computer sciences, recruited through an online experimental participant volunteer panel on the School of Psychology's web pages. They volunteered to take part in what was described as a "decision making experiment," earning an average of $£ 6.20$ (about $\$ 12$ ) each, range $£ 4.80$ to $£ 8.40$, depending on the points that they scored in 12 games, six of which were excluded from analysis for reasons explained in the Results section below. The sample included 36 men and 36 women, with ages ranging from 18 to 31 years and a mean of $20.50(S D=2.16)$ years.

\section{Design}

A randomized-groups experimental design was used, with players assigned to the following three treatment conditions. In the first (phantom decoy) condition, players made one-off decisions in six symmetric $3 \times 3$ games in which each player invariably had one strategy strictly dominating just one other, in the sense of yielding a strictly higher payoff irrespective of the coplayer's choice, but the dominated strategies were phantom decoys, visible but unavailable for choice. A second (dominated-available) condition was similar to the phantom decoy condition, except that the asymmetrically dominated strategies were available for choice. In a third (control) condition, players made one-off decisions in six $2 \times 2$ games constructed by deleting the asymmetrically dominated row and column from each of the $3 \times 3$ games.

From a strategic point of view, the phantom decoy and control conditions were identical, apart from the strategically irrelevant context provided by the unavailable third strategy in the phantom decoy condition, and any difference in choice behavior between these two conditions must be interpreted as a context effect. In a meaningful sense, the dominated-available condition was also strategically equivalent to the phantom decoy and control conditions, for players who are fully rational in the game-theoretic sense and have common knowledge of this rationality, because strictly dominated strategies are inadmissible for rational players, who never choose them and know that their co-players never choose them.

\section{Materials}

The six $3 \times 3$ games used in Experiment 1 (shown in Appendix B) were based on symmetric $2 \times 2$ games, all but two of which (Games 1 and 4) lacked dominant strategies. An example of a game used in Experiment 1 is shown in Figure 1: this game elicited the largest asymmetric dominance effect. The dominant strategies are shown in row and column $C$ and the dominated 
strategies in row and column $E$, although in the experiment, to control for positioning and labeling effects, strategies were rotated systematically so that they appeared equally frequently in all three rows and columns. We refer to the asymmetrically dominant strategy as $C$ and the dominated strategy as $E$, although they were not invariably labeled as such in the payoff matrices seen by the players. To aid comprehension, the games were presented to the players with labeling and payoffs in red for Player I and in blue for Player II, and each cell was divided diagonally from top-left to bottom-right to make the payoff matrices easier to read. The payoffs represented cash in units of pence sterling, ranging from zero to 80 pence in each game.

The main dependent variable was the relative frequency of $C$ strategy choices - choices of asymmetrically dominant strategies or their counterparts in the $2 \times 2$ versions of the games, not necessarily labeled $C$ in the versions presented to the players. A secondary dependent variable was the players' self-rated confidence in their decisions.

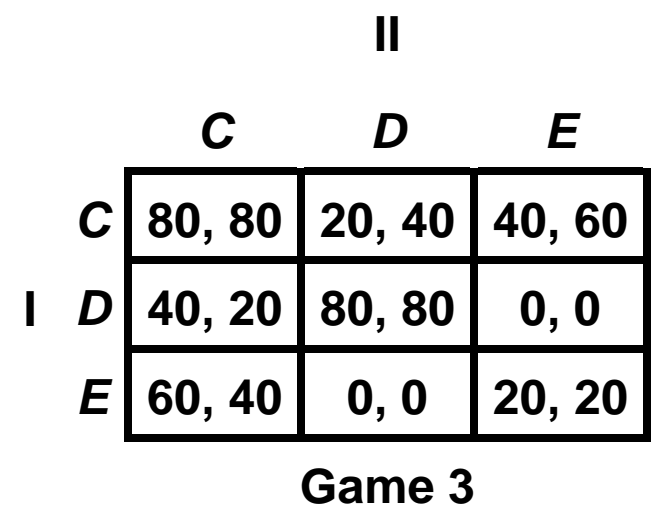

Figure 1. One of the games used in Experiment 1. Player I chooses between rows $C, D$, and $E$, Player II chooses between the columns, and the payoffs to Players I and II are shown in that order in the cell corresponding to the players' choices. Strategy $C$ strictly dominates Strategy $E$, because it yields a strictly higher payoff whichever strategy the co-player chooses.

Players indicated their strategy choices by circling $C, D$, or $E$ on answer sheets in the dominated-available treatment condition, or by circling $C$ or $D$ in the control condition from which $E$ was omitted. In the phantom decoy condition, players were shown $3 \times 3$ matrices, as in the dominated-available condition, but after studying each matrix, when they came to make their choices, they found that only two options were available on the answer sheet, because $E$ was always unavailable, as it was the phantom decoy (sometimes labeled $C$ or $D$ on the players' materials). The answer sheets also included small spaces in which players could briefly summarize the reasons for their choices.

Finally, for each choice, players responded to a Likert scale ranging from 0 to 10, anchored by the phrases not at all confident and totally confident respectively, indicating how confident they felt that the choice they made was the best one available.

\section{Procedure}

Pairs of participants were allocated quasi-randomly to treatment conditions, maintaining equal numbers of male-male, female-female, and male-female pairs in each treatment condition to control for gender effects. However, no significant gender effects emerged, and we shall say nothing more about this variable. 
Between one and four pairs were tested in each experimental session. Players were seated so that they could not see their co-players, and communication between players was forbidden. After they had finished reading a page of instructions and filling in demographic information on their answer sheets, the experimenters conducted question-and-answer sessions, using three sample payoff matrices similar to those used in the appropriate treatment condition to clarify the players' understanding of the matrices. It was explained to players at that point that they and their co-players would be looking at exactly the same payoff matrix on each trial.

In the phantom decoy treatment condition, in which each payoff matrix included a strategy that could not be chosen, players were told:

One of the rows and one of the columns is not available and can't be chosen by either person. You will not know which one is unavailable until you turn over the answer sheet for that problem. If, for example, you decide to choose row $C$ and have found that this option is unavailable on the answer sheet, please go back to the payoff diagrams and make another choice.

The unavailable strategies were always the strictly dominated $E$ strategies (though not always labeled as such in the materials), and we did not expect players to choose them, except by mistake or through misunderstanding. The players recorded their choices, reasons, and confidence ratings for all six matrices during experimental sessions lasting approximately 35 minutes. They were not provided with any feedback until they had completed all of the games. At the end of each session, the experimenters added up the players' scores, taking into account their choices and those of their co-players, and paid them whatever they had earned.

\section{Results}

In the dominated-available treatment condition, in which the dominated Strategy $E$ was available for choice, only two players ever chose it. One chose it once out of six games and another chose it twice. The relative frequency of dominated strategy choices was thus 0.69 per cent, suggesting that, in the main, the players understood the payoff matrices and the dominance relation that was crucial to the experiment. This was corroborated by their stated reasons for their choices. These comments were generally reasonable and thoughtful, and when a dominant strategy was present, they often alluded to the fact that a particular option was better (or worse) than another whatever the other player might choose.

\section{C choices}

The main dependent variable was the relative frequency with which players chose the asymmetrically dominant strategies and their counterparts in the $2 \times 2$ versions of the games from which the dominated strategies were deleted. The mean numbers of $C$ choices across treatment conditions are shown in Table 1.

Table 1. Experiment 1: Mean numbers of $C$ choices across treatment conditions

\begin{tabular}{lccc}
\hline & $N$ & Mean & $S D$ \\
\hline Phantom decoy & 24 & 2.63 & 1.43 \\
Dominated-available & 24 & 3.25 & 1.42 \\
Control & 24 & 1.50 & .83 \\
Total & 72 & 2.46 & 1.44 \\
\hline
\end{tabular}


The mean relative frequency of $C$ choices in the control condition, without asymmetric dominance, was 1.50 times out of six, or 25.00 per cent, compared to 43.83 per cent in the phantom decoy condition and 54.17 per cent in the dominated-available condition. The differences between these three means are highly significant: $F(2,69)=11.826, p<.0001$, partial $\eta^{2}=.26$, suggesting that a substantial proportion of the variance in $C$ choices is explained by differences between treatment conditions. Post-hoc multiple comparisons using the TukeyHSD procedure revealed that the phantom decoy and dominated-available means do not differ significantly from each other, but that the phantom decoy mean is significantly higher than the control group mean $(p<.008)$, and the dominated-available mean is also significantly higher than the control group mean $(p<.0001) .^{2}$ These findings corroborate Hypotheses 1 and 2 .

\section{Game-by-game analysis}

Table 2 shows the relative frequencies of $C$ choices across treatment conditions, separately for each game, with chi-square values, associated probability levels, and values of the effect size statistic $w$. According to Cohen (1992), an effect is small if $w \geq .10$, medium if $w \geq .30$, and large if $w \geq .50$. The figures in the columns labeled PD (phantom decoy), DA (dominated-available), and ND (control) are shown as percentages, although the chi-square values were calculated from raw frequencies. Four of the six games exhibited the strategic asymmetric dominance effect significantly, the exceptions being Games 2 and 5. In the control condition, the percentages of $C$ choices were quite low in both of these games (29 percent and 25 per cent respectively), so that there was no ceiling to prevent the effect from occurring. Probable explanations for these anomalies will be addressed in the Discussion section below.

Table 2. Experiment 1: Percentages of $C$ choices across phantom decoy (PD), dominatedavailable (DA), and control (ND) treatment conditions in each of six games, with chi-square test results and effect sizes $w$

\begin{tabular}{ccrrrll}
\hline Game & PD & DA & ND & $\chi^{2}$ & $p$ & $w$ \\
\hline 1 & 42 & 50 & 17 & 6.26 & .04 & .29 \\
2 & 21 & 17 & 29 & 1.13 & .57 & .13 \\
3 & 71 & 83 & 8 & 31.22 & .01 & .66 \\
4 & 42 & 67 & 29 & 7.05 & .03 & .31 \\
5 & 21 & 29 & 25 & 0.44 & .80 & .08 \\
6 & 67 & 79 & 42 & 7.47 & .02 & .32 \\
\hline
\end{tabular}

\section{Confidence ratings}

A 2 (treatment condition) $\times 2$ (dominant or non-dominant strategy chosen) ANOVA $^{3}$ revealed that players' self-rated confidence in their decisions, on a scale from 0 to 10 , differed significantly across treatment conditions. In the phantom decoy condition, the mean was $M=$ $7.34, S D=1.22$; in the dominated-available condition, $M=6.76, S D=1.60$; and in the $2 \times 2$ control condition, $M=6.03, S D=1.64$. These means differ significantly, $F(2,58)=5.98, p=$ .004 , partial $\eta^{2}=.17$. Post-hoc Tukey-HSD multiple comparisons revealed that the first two means do not differ significantly from each other $(p=.27)$, but confidence was significantly higher in the presence of an asymmetrically dominated strategy in the phantom decoy than in the control condition $(p=.003)$. The dominated-available and control condition did not significantly differ $(p=.14)$. 
Table 3. Experiment 1: Mean confidence ratings in $C$ and $D$ choices across phantom decoy (PD), dominated-available (DA), and control (ND) treatment conditions in each of the six games

\begin{tabular}{cccccccc}
\hline & \multicolumn{3}{c}{ C choices } & & \multicolumn{3}{c}{$D$ choices } \\
\cline { 2 - 4 } \cline { 6 - 8 } Game & PD & DA & ND & & PD & DA & ND \\
\hline 1 & 5.90 & 5.67 & 5.33 & & 6.57 & 6.00 & 6.15 \\
2 & 7.40 & 7.50 & 6.71 & & 8.37 & 7.79 & 7.18 \\
3 & 7.76 & 7.05 & 5.00 & & 6.86 & 6.50 & 6.09 \\
4 & 7.33 & 6.06 & 5.00 & & 6.86 & 5.63 & 6.88 \\
5 & 7.20 & 6.14 & 5.40 & & 8.95 & 8.18 & 7.50 \\
6 & 7.40 & 7.67 & 4.20 & & 7.50 & 5.50 & 7.43 \\
Mean & 7.17 & 6.56 & 5.25 & & 7.51 & 6.96 & 6.80 \\
\hline
\end{tabular}

Overall, participants were less confident when choosing $C(M=6.36, S D=1.77)$ than when choosing the $D$ strategy $(M=7.10, S D=1.44), F(1,58)=10.11, p=.002$, partial $\eta^{2}=.15$. The explanation for this can be found by considering the interaction of treatment condition and chosen strategy, $F(2,58)=2.65, p=.08$, partial $\eta^{2}=.08$, and the game-by-game confidence ratings, broken down by treatment condition and choice of strategy in Table 3. Participants tended to choose $D$ in the control condition and were more confident in that strategy $(M=6.80$, $S D=1.14)$ than when choosing the $C$ strategy $(M=5.25, S D=2.15)$. Introducing the dominated strategy caused both an increase in choosing $C$ (as previously discussed) and a significant increase in the confidence felt in the $C$ strategy in both the phantom decoy condition $(M=7.17$, $S D=1.12)$ and in the dominated-available condition $(M=6.56, S D=1.46)$ compared with the control condition $(M=5.25, S D=2.15), F(2,58)=7.39, p=.001$, partial $\eta^{2}=.20$, (post- hoc Tukey-HSD tests reveal that the first two conditions do not differ from each other $p=.44$, but both are higher than the control, $p=.001$ and $p=.033$ respectively). There was no significant increase in confidence when choosing the $D$ strategy in any treatment condition (see Table 3 ), $F(2,58)=1.36, p=.26$, ns, partial $\eta^{2}=.05$, presumably because participants were not choosing the strategy that dominated $E$, and thus their confidence did not increase.

\section{Discussion}

The results of Experiment 1 corroborate our main Hypotheses 1 and 2. Dominant $C$ strategies were chosen significantly more frequently in the $3 \times 3$ versions of the games, and this effect was significant even when the dominated strategies were phantom decoys, replicating a finding on asymmetric dominance in individual choice (Doyle, O'Connor, Reynolds, \& Bottomley, 1999).

The game-by-game analysis in Table 2 sheds more light on the results of Experiment 1. Most of the games exhibited the asymmetric dominance effect, the frequencies of $C$ choices being significantly higher in the phantom decoy and dominated-available conditions than in the nodominance control condition. The exceptions were Games 2 and 5, and it is not difficult to explain why these games did not follow the predicted pattern. A property that they share in common is the payoff dominance of the $(D, D)$ Nash equilibrium. Although neither player has a dominant strategy in either of the $2 \times 2$ versions of the games, both $2 \times 2$ games have Nash equilibria at $(D, D)$ and $(C, C)$, and payoffs are higher for both players in the $(D, D)$ than the $(C$, $C$ ) equilibrium. Selection of payoff-dominant equilibria is natural and compelling (Bacharach, 1999; Colman, 2003; Colman \& Bacharach, 1997; Colman \& Stirk, 1998; Cooper, et al., 1990; Harsanyi \& Selten, 1988; Sugden, 1993). In Games 2 and 5, the payoff dominance of the $(D, D)$ outcome may have counteracted the influence of the strategic dominance of $C$ over $E$ to some extent in the $3 \times 3$ versions of these games by providing players with a persuasive reason for 
choosing $D$. None of the other games had payoff-dominant equilibria. These findings are consistent with the interpretation that payoff-dominant outcomes function as focal points (in the sense of Schelling, 1960; see also Janssen, 2006; Sugden \& Zamarrón, 2006) for coordinating actions and expectations. The asymmetric dominance effect seems most likely to emerge when the control version of a game lacks a focal point on which players might expect to coordinate, presumably because asymmetric dominance provides a reason for choice, but it can be overwhelmed by a strong focal point such as a payoff-dominant equilibrium that offers an alternative reason for choice.

Game 3 also merits comment, because it elicited the largest and most highly significant asymmetric dominance effect. The reason was almost certainly the exceptionally low relative frequency of $C$ choices in the $2 \times 2$ control condition. This low base of 8 per cent $C$ choicesconsiderably lower than any other game used in the experiment - left more scope than other games for increased $C$ choices in the phantom decoy and dominated-available conditions, in which the $C$ strategies dominated $E$ strategies.

Self-rated decision confidence was significantly higher when asymmetrically dominant strategies were present than in the control condition, and this effect was significant even in the phantom decoy condition, in which the dominated strategies could not be chosen. We shall comment in the General Discussion on a possible explanation for this confidence-bolstering effect.

\section{Experiment 2}

The results of Experiment 1 provide some evidence for asymmetric dominance and phantom decoy effects in games, but the results, though significant, rest on a relatively small number of symmetric games. A new set of 12 games based on asymmetric $2 \times 2$ games was therefore devised for Experiment 2, partly to provide a broader-based test of the main hypotheses, and partly because suitable strategic structures for symmetric $2 \times 2$ games had been exhausted in Experiment 1.

\section{Participants}

The participants were 81 undergraduate students recruited through the same online participant panel as was used in Experiment 1 but excluding anyone who had participated in that experiment. The sample comprised 30 men and 51 women, with ages ranging from 17 to 50 years and a mean of $22.34(S D=5.83)$ years. In this experiment, participants earned an average of $£ 7.20$ (about \$14) each, range $£ 5.00$ to $£ 9.40$, depending on the points scored in the games. Design and Materials

A randomized-groups experimental design was used, similar to the design used in Experiment 1. The 12 games used in Experiment 2 (shown in Appendix B) were based on $2 \times 2$ games chosen from the taxonomy of Rapoport, Guyer, and Gordon (1976). We selected $2 \times 2$ mixed-motive games without dominant strategies and without unique or payoff-dominant Nash equilibria (ignoring mixed strategies involving randomization). A Nash equilibrium is a combination of players' strategies in which each player makes a best reply to the co-player's strategy, a best reply being a strategy yielding the highest payoff given the co-player's strategy choice. If a game has a unique Nash equilibrium, then rational players are bound to choose it, according to the assumptions of game theory. If a game has more than one Nash equilibrium, but one is payoff-dominant in the sense that it yields both players a higher payoff than the other, then that is the "natural" outcome of the game and, once again, we should expect rational players to choose it (Harsanyi \& Selten, 1988). Our selection of 12 games without unique or payoff- 
dominant pure-strategy equilibria was intended to maximize the scope for asymmetric dominance effects.

We constructed $3 \times 3$ versions of these games by first identifying the strategies that were predominantly dispreferred by players in an unpublished empirical study of single plays in $2 \times 2$ games carried out by Frenkel and reported in Rapoport, Guyer, and Gordon (1976, pp. 135-150). We labeled these strategies $C$ and then added third strategies, asymmetrically dominated by them, which we labeled $E$, to the games. An example of one of the games used in Experiment 2 - the one that yielded the largest asymmetric dominance effect-is shown in Figure 2. This game has Nash equilibria at $(C, C)$ and $(D, D)$, but neither of these is payoff dominant, because nether yields a higher payoff to both players than the other. Strategy $C$ dominates Strategy $E$ for both players and, as in all our games, there are no other dominance relations between strategies.

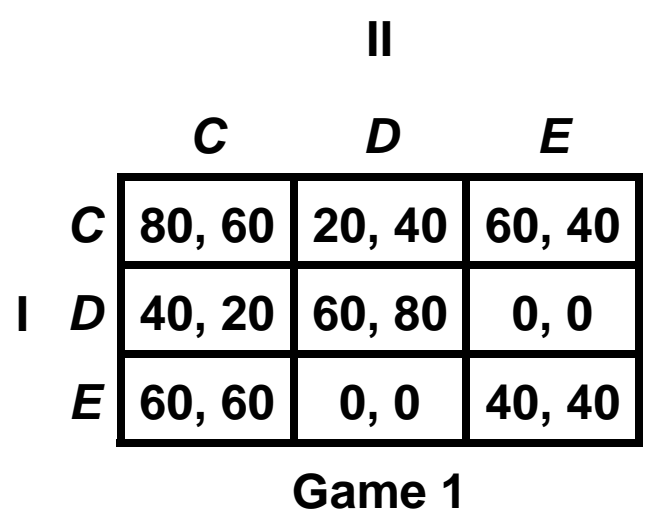

Figure 2. An asymmetric game used in Experiment 2, with no dominance relation between Strategies $C$ and $D$. Strategy $C$ strictly dominates Strategy $E$, because it yields a higher payoff irrespective of the co-player's strategy choice.

The payoff matrices were presented to the participants as in Experiment 1, color-coded as an aid to comprehension, and once again the payoffs represented cash values in units of pence sterling, ranging from 0 pence to 80 pence. Rows and columns were systematically rotated to control for positioning and labeling effects although, once again for convenience, we refer to the dominant strategy as $C$ and the dominated strategy as $E$. The dependent variables were the relative frequencies of $C$ choices (not necessarily labeled $C$ in the versions presented to the players) and the players' self-rated confidence in their decisions.

In this experiment, to control for extraneous sources of variance arising from the fact that the games were asymmetric, participants were all assigned to the role of Player I, and one additional participant served as Player II to enable meaningful payoffs to be calculated (this player's choices were not included in the analysis below). Participants were told truthfully that they would each be paired with another player but, in fact, there was only one Player II. ${ }^{4}$

\section{Procedure}

The procedure was similar to Experiment 1, apart from the use of asymmetric games. The instructions given to the participants were essentially the same, but players were randomly assigned to treatment conditions without regard to gender. In light of the absence of gender differences in Experiment 1, we did not examine this variable again in Experiment 2. Players made one-off strategy choices in all 12 games during experimental testing sessions that lasted approximately 40 minutes. No feedback was given until they had completed all 12 games. 


\section{Results}

In the dominated-available treatment condition, in which the dominated strategy $E$ was available for choice, players chose it rarely. One participant chose $E$ eight times, one chose it six times, one chose it four times, two chose it twice, and two chose it once, yielding an overall relative frequency of dominated strategy choice of 2.47 per cent. Although dominated strategy choices were slightly more frequent than in Experiment 1, presumably because the asymmetric payoff matrices were more complex and harder to analyze, these data suggest that most players understood the games, including the crucial dominance property.

C choices

The relative frequencies with which players chose the asymmetrically dominant $C$ strategies and their counterparts in the $2 \times 2$ versions of the games are shown in Table 4 . Differences between these three means are significant, $F(2,78)=6.44, p=.01$, partial $\eta^{2}=.14$. Post-hoc multiple comparisons using the Tukey-HSD procedure reveal that the means for the phantom decoy and dominated-available treatment conditions are not significantly different from each other but that both are significantly larger than the mean for the control condition $(p<.05)$, confirming the major experimental hypotheses that players would choose $C$ significantly more frequently when it was asymmetrically dominant, both when the $E$ strategy that it dominated was available for choice (Hypothesis 1) and when it was a phantom decoy (Hypothesis 2).

Table 4. Experiment 2: Mean numbers of $\mathrm{C}$ choices (out of 12) across treatment conditions

\begin{tabular}{llll}
\hline & $N$ & Mean & $S D$ \\
\hline Phantom decoy & 27 & 7.93 & 1.57 \\
Dominated-available & 27 & 7.81 & 2.18 \\
Control & 27 & 6.37 & 1.50 \\
Total & 81 & 7.37 & 1.89 \\
\hline
\end{tabular}

\section{Game-by-game analysis}

In Table 5, the relative frequencies of $C$ choices across treatment conditions are shown separately for each game, together with chi-square values, significance levels and values of the effect size statistic $w$. The asymmetric dominance effect is significant in the hypothesized direction (that is, with the smallest mean in the no-dominance control condition) in six of the games. In addition, in Game 9, the percentages differ in the hypothesized direction and there is a medium effect size, but the effect is only marginally significant. In two of the games in which the effect was not found, a ceiling effect is evident. In Game 5, for example, 89 per cent of players chose $C$ in the $2 \times 2$ control condition, and in Game 12, 70 per cent of them chose it in the control condition. These were the two games with the highest frequencies of $C$ choices in the control condition, and in both cases, relatively little headroom was left to increase the frequency of $C$ choices when a dominated $E$ strategy was appended in the phantom decoy and dominatedavailable conditions. 
Table 5. Experiment 2: Percentages of $C$ choices across phantom decoy (PD), dominatedavailable (DA), and control (ND) treatment conditions in each of 12 games, with chi-square test results and effect sizes $w$

\begin{tabular}{cccccll}
\hline Game & PD & DA & ND & $\chi^{2}$ & $p$ & $w$ \\
\hline 1 & 44 & 56 & 26 & 22.25 & .000 & .52 \\
2 & 74 & 96 & 56 & 12.08 & .002 & .39 \\
3 & 78 & 85 & 67 & 12.74 & .01 & .40 \\
4 & 78 & 78 & 44 & 9.00 & .01 & .33 \\
5 & 82 & 82 & 89 & 0.73 & .69 & .09 \\
6 & 59 & 44 & 59 & 6.79 & .15 & .29 \\
7 & 74 & 70 & 56 & 13.04 & .01 & .40 \\
8 & 78 & 44 & 41 & 14.61 & .01 & .42 \\
9 & 37 & 52 & 22 & 7.64 & .11 & .31 \\
10 & 74 & 56 & 59 & 7.71 & .10 & .31 \\
11 & 44 & 56 & 48 & 0.69 & .71 & .09 \\
12 & 70 & 63 & 70 & 2.23 & .69 & .17 \\
\hline
\end{tabular}

\section{Confidence ratings}

The same analyses as for Experiment 1 were carried out on confidence ratings. Mean confidence ratings on a scale from 0 to 10 were highest in the phantom decoy condition $(M=$ $6.57, S D=1.53)$. The means in the dominated-available condition $(M=5.65, S D=1.80)$ and the control condition $(M=5.90, S D=1.09)$ were slightly lower. The three means differ significantly, $F(2,78)=3.18, p=.05$, partial $\eta^{2}=.08$, and post-hoc Tukey-HSD multiple comparisons confirm that confidence was significantly higher in the phantom decoy condition than in the dominated-available condition $(p=.05)$ and that other differences were nonsignificant.

Furthermore, confidence was higher when choosing dominant $C$ strategies $(M=6.27, S D=1.46)$ than non-dominant $D$ strategies $(M=5.81, S D=1.61)$, and this difference is significant, $F(1,78)$ $=13.70, p<.001$, partial $\eta^{2}=.15$, providing further support for the suggestion that asymmetric dominance bolsters decision confidence. Game-by-game confidence ratings, broken down by treatment condition and choice of strategy, are shown in Table 6. 
Table 6. Experiment 2: Mean confidence ratings in $C$ and $D$ choices across phantom decoy (PD), dominated-available (DA), and control (ND) treatment conditions in each of the 12 games

\begin{tabular}{|c|c|c|c|c|c|c|}
\hline \multirow[b]{2}{*}{ Game } & \multicolumn{3}{|c|}{$C$ choices } & \multicolumn{3}{|c|}{$D$ choices } \\
\hline & PD & DA & ND & PD & DA & ND \\
\hline 1 & 6.75 & 6.27 & 5.86 & 6.87 & 6.45 & 5.70 \\
\hline 2 & 6.50 & 6.35 & 5.53 & 5.00 & 4.00 & 6.00 \\
\hline 3 & 7.29 & 5.70 & 6.22 & 6.83 & 7.00 & 5.33 \\
\hline 4 & 6.90 & 6.29 & 5.58 & 8.00 & 6.00 & 6.53 \\
\hline 5 & 7.50 & 6.18 & 6.74 & 5.40 & 5.20 & 7.00 \\
\hline 6 & 6.31 & 5.50 & 5.56 & 5.64 & 5.33 & 5.36 \\
\hline 7 & 6.00 & 5.95 & 5.73 & 6.14 & 5.88 & 6.25 \\
\hline 8 & 6.65 & 5.25 & 4.91 & 6.50 & 6.27 & 5.44 \\
\hline 9 & 7.40 & 6.71 & 5.50 & 6.82 & 4.92 & 5.81 \\
\hline 10 & 6.35 & 4.80 & 5.50 & 4.33 & 5.58 & 5.18 \\
\hline 11 & 6.73 & 6.53 & 6.46 & 7.07 & 4.42 & 6.50 \\
\hline 12 & 6.11 & 5.24 & 6.53 & 6.88 & 6.60 & 4.63 \\
\hline Mean & 6.74 & 6.03 & 6.04 & 6.40 & 5.27 & 5.75 \\
\hline
\end{tabular}

The overall means hide the pattern of results somewhat, but by examining Table 6 with the results shown in Table 5 in mind it is clear that in the six games in which asymmetric dominance occurred $(1,2,3,4,7$, and 8$)$, confidence when choosing the dominating strategy $C$ was higher in the phantom decoy and dominated-available conditions than in the control condition, following the same pattern as shown in Experiment 1.

\section{General Discussion}

We capitalized on the game-theoretic concept of strategic dominance to define a form of asymmetric dominance applicable to interactive decisions and to investigate asymmetric dominance and phantom decoy effects in experimental games. Strategic dominance is a feature of any game in which a player has one strategy that dominates another, and a strategic asymmetric dominance effect may be said to occur if the addition of a dominated strategy to a game without dominance relations increases players' preferences for the dominating strategy relative to a strategy that is neither dominant nor dominated. We proved that the asymmetric dominance effect violates Sen's (1969) Property $\alpha$ and hence also the first postulate in Savage's 
(1954/1972) subjective expected utility (SEU) theory, his most fundamental axiom of rational choice. The principal aim of the experiments reported in this article was to seek empirical evidence for the occurrence of strategic asymmetric dominance and phantom decoy effects in games.

In Experiment 1, $C$ strategies were chosen more frequently in the treatment condition in which they asymmetrically dominated third strategies than in the control condition without dominated strategies (see Tables 1 and 2). Dominant $C$ strategies were chosen significantly more frequently in the $3 \times 3$ versions of the games, even when the dominated strategies were phantom decoys. These findings corroborate our main experimental Hypotheses 1 and 2.

The effects were highly significant, with substantial effect sizes. Compared to the control condition, the effect occurred not only in the dominated-available condition but also in the phantom decoy condition in which the dominated strategy was visible but unavailable for choice. It is worth noting that in the phantom decoy condition, the games were strategically identical to those in the $2 \times 2$ control condition, from which the dominated strategies were deleted, differing only in extraneous context provided by the unavailable dominated strategies, yet for these games the effect was only slightly smaller than in the dominated-available condition. This corroborates and extends the findings of Doyle, O'Connor, Reynolds, and Bottomley (1999) and others regarding asymmetrically dominated phantom decoys in individual decision making. In the dominated-available condition, the $3 \times 3$ versions were strategically equivalent to their $2 \times 2$ counterparts for game-theoretically rational players for whom strictly dominated strategies are inadmissible, assuming that the players' rationality is common knowledge in the game-each player knows it, knows that the co-player knows it, and so on.

In Experiment 2, we used asymmetric games without unique or payoff-dominant purestrategy equilibria. Significantly more players chose the $C$ strategy when it dominated a third $E$ strategy, both in the phantom decoy condition, where $E$ was visible but unavailable for choice, and in the dominated-available condition, where $E$ could be chosen. These findings provide further corroboration of Hypotheses 1 and 2. When data from the 12 games used in Experiment 2 were analyzed separately (Table 5), this asymmetric dominance effect turned out to be statistically significant in six of the games. In a seventh (Game 9) the $C$ strategy was chosen less frequently in the control condition than in the dominated-available or phantom decoy conditions, in line with Hypotheses 1 and 2 respectively, but the effect was only marginally significant. Two games that failed to elicit the effect (Games 5 and 12) were the two with the highest frequencies of $C$ choices in the $2 \times 2$ control condition, and this is strongly suggestive of ceiling effects limiting the possibility of increasing $C$ choices when they became dominant in the phantom decoy and dominated-available conditions.

In all of the games used in Experiment 2, the $C$ strategies were the ones that were predominantly dispreferred by players in Frenkel's study (reported in Rapoport, Guyer, \& Gordon, 1976, pp. 135-150) of single plays in $2 \times 2$ games. We deliberately chose these strategies to avoid ceiling effects when their attractiveness was increased by making them dominate third strategies. In a few cases, the $C$ strategies were nonetheless preferred by our players in the $2 \times 2$ control condition versions. The reasons for the differences between preferences in our Experiment 2 and in Frenkel's study are conjectural, but there are at least three possibilities. First, the players in Experiment 2 were male and female British undergraduate students, whereas Frenkel's players were all male Canadian undergraduates, and there is evidence (reviewed in Colman, 1995 and elsewhere) to show that both sex and nationality often influence choices in mixed-motive games, although it is worth recalling that no sex differences were found in Experiment 1. Second, the payoffs in each of the games used in Experiment 2 were $0,20,40,60$, or 80 and represented monetary payoffs in pence sterling, whereas in 
Frankel's study they were $-10,-1,1$, or 10 and did not represent money: prizes of $\$ 15, \$ 10$, and $\$ 5$ were offered to the three participants among 96 who accumulated the largest total points over all the games. Third, the players in Experiment 2 made 12 individual decisions in a single 40minute session, whereas Frenkel's players made 320 decisions (78 different games, each repeated at least twice) in their own time over a period of 48 hours and were told that they could consult with others if they wished.

Analysis of players' self-rated confidence in their decisions in Experiment 1 revealed evidence of significantly higher confidence in the chosen strategy when it dominated an inferior strategy, even if that strategy was not available for choice. This tends to suggest that the asymmetric dominance and phantom decoy effects may be due to a bolstering of confidence in strategies when they have other strategies to dominate. In any individual or interactive choice, an alternative tends to appear more attractive when juxtaposed with something manifestly less attractive on all relevant attributes or dimensions, and our evidence suggests that this increases decision makers' confidence to choose it, in line with reason-based choice theory.

In Experiment 2, self-rated decision confidence was significantly higher in the phantom decoy condition than in the dominated-available condition. This suggests that the presence of phantom decoy strategies bolstered players' decision confidence more than asymmetrically dominated strategies that were available for choice. The most obvious reason is that players had only two available strategies from which to choose in the phantom decoy condition, and are therefore likely, other things being equal, to have more confidence in their choices than in the dominated-available condition, in which they chose from three available strategies. It is also possible that players processed the payoff matrices more deeply in the phantom decoy than in the dominated-available condition, because they knew that one of their strategies would turn out to be unavailable for choice, and this may have contributed to their greater decision confidence in the phantom decoy condition. Confidence was also significantly higher for players choosing dominant $C$ strategies than nondominant $D$ strategies, providing further support for the suggestion that asymmetric dominance bolsters decision confidence.

Our data support an interpretation of asymmetric dominance and phantom decoy effects in terms of confidence-bolstering effects of reasons for choice. The presence of an asymmetrically dominated or phantom decoy strategy appears to bolster players' confidence in choosing the strategy that dominates it, presumably because a strategy appears more choiceworthy when it can be seen to be unambiguously better than another, even if the other strategy is obviously the worst option and therefore not strictly relevant to the choice between the other two strategies. This confidence-bolstering effect may also explain asymmetric dominance and phantom decoy effects in individual decision making.

The cognitive mechanism underlying this form of confidence-bolstering may be reason-based choice. Tversky and Shafir (1992) first proposed that people tend to look for compelling reasons when there are no obvious rules or evaluations to guide difficult decisions. They described an experiment in which participants imagined they had just taken a tough qualifying examination. Members of one group were told they had passed the exam, others that they had failed, and a third group were told that they would learn the results the following day. Members of each group were invited to choose between buying a vacation in Hawaii immediately, not buying the vacation, or paying $\$ 5$ to retain the right to buy the vacation the following day. The majority of those who had purportedly passed or had failed the examination chose to buy the vacation, but the majority of those who did not know the examination's outcome chose to retain the right to buy the vacation the following day, presumably because they did not have a compelling reason to buy it while the exam's outcome was unknown. Several other examples of the same phenomenon were quickly discovered (Shafir, Simonson, \& Tversky, 1993; Shafir \& Tversky, 1992). 
Something similar may help to explain asymmetric dominance effects. The addition of a dominated strategy to an existing set of strategies provides a cogent reason for choosing the dominant strategy in a game in which there are no other obvious reasons to prefer any strategy. The availability of a reason in an otherwise obscure and difficult decision task is also likely to boost the confidence of decision makers to choose asymmetrically dominant options or strategies.

Players' confidence ratings in Experiments 1 and 2 do not fit reason-based choice theory perfectly, but we should perhaps not expect a perfect fit with such a crude measure as self-rated confidence. In most cases, confidence was significantly higher in phantom decoy and dominatedavailable than control conditions, as predicted by reason-based choice theory, and in some cases these differences were nonsignificant; however, there were no significant differences in the opposite direction. Other factors may help to explain asymmetric dominance and phantom decoy effects. Cooper et al. (1990) provided evidence that an additional dominated strategy can function as a coordinating device in a $2 \times 2$ coordination game with multiple Nash equilibria, guiding players toward the same equilibrium. This may have occurred in some of our games, but it cannot provide a complete explanation, because only a few of our $2 \times 2$ games had multiple equilibria.

Taken together, the results of Experiments 1 and 2 provide strong evidence for the existence of an asymmetric dominance and phantom decoy effects in dyadic games without focal points on which players can coordinate their actions and expectations of their co-players' actions. In Experiment 1, the size of the asymmetric dominance effect was partial $\eta^{2}=.26$, and in Experiment 2 it was partial $\eta^{2}=.14$, and according to the threshold suggested by Cohen (1988), both of these should be interpreted as large effect sizes. The effect does not occur in all gamesit can apparently be suppressed by ceiling effects and overwhelmed by payoff dominance-but it appears to be reasonably robust across a wide range of symmetric and asymmetric games without these properties. In line with findings from experiments on individual decision making (e.g., Ariely \& Wallsten, 1995; Doyle, O'Connor, Reynolds, \& Bottomley, 1999; Mishra, Umesh, \& Stem, 1993), it can occur even as a mere context effect, when the dominated strategy is a phantom decoy that cannot be chosen. Asymmetric dominance is a real possibility in strategic interactions in interpersonal, economic, military, and political contexts, whenever a player has to choose between two or more equally attractive options and can see no obvious reason to prefer one rather than another, apart from the fact that just one of them is clearly preferable to a less attractive option in all circumstances that might arise.

\section{References}

Ariely, D., \& Wallsten, T. S. (1995). Seeking subjective dominance in multi-dimensional space: An explanation of the asymmetric dominance effect. Organizational Behavior and Human Decision Processes, 63, 223-232.

Bacharach, M. (1999). Interactive team reasoning: A contribution to the theory of co-operation. Research in Economics, 53, 117-147.

Bastardi, A., \& Shafir, E. (1998). On the pursuit and misuse of useless information. Journal of Personality and Social Psychology, 75, 19-32.

Carpenter, G. S., Glazer, R., \& Nakamoto, K. (1994). Meaningful brands from meaningless differentiation: The dependence on irrelevant attributes. Journal of Marketing Research, 31, $339-350$.

Chernoff, H. (1954). Rational selection of decision functions. Econometrica, 22, 423-443.

Cohen, J. (1988). Statistical power analysis for the behavioral sciences (2nd ed.). Hillsdale, NJ: Erlbaum. 
Cohen, J. (1992). A power primer. Psychological Bulletin, 112, 155-159.

Colman, A. M. (1995). Game theory and its applications in the social and biological sciences (2nd ed.). London: Routledge.

Colman, A. M. (2003). Cooperation, psychological game theory, and limitations of rationality in social interaction. Behavioral and Brain Sciences, 26, 139-153.

Colman, A. M., \& Bacharach, M. (1997). Payoff dominance and the Stackelberg heuristic. Theory and Decision, 43, 1-19.

Colman, A. M., \& Stirk, J. A. (1998). Stackelberg reasoning in mixed-motive games: An experimental investigation. Journal of Economic Psychology, 19, 279-293.

Cooper, R. W., DeJong, D. V., Forsythe, R., \& Ross, T. W. (1990). Selection criteria in coordination games: Some experimental results. American Economic Review, 80, 218-233.

Doyle, J. R., O’Connor, D. J., Reynolds, G. M., \& Bottomley, P. A. (1999). The robustness of the asymmetrically dominated effect: Buying frames, phantom alternatives, and in-store purchases. Psychology and Marketing, 16, 225-243.

Fishburn, P. C. (1973). The theory of social choice. Princeton, NJ: Princeton University Press.

Harsanyi, J. C., \& Selten, R. (1988). A general theory of equilibrium selection in games. Cambridge, MA: MIT Press.

Huber, J., Payne, J. W., \& Puto, C. (1982). Adding asymmetrically dominated alternatives: Violations of regularity and the similarity hypothesis. Journal of Consumer Research, 9, 9098.

Janssen, M. C. W. (2006). On the strategic use of focal points in bargaining situations. Journal of Economic Psychology, 27, 622-634.

Kreps, D. M. (1988). Notes on the theory of choice. Boulder, CO: Westview.

Luce, D. (1959). Individual choice behavior. New York: Wiley.

Luce, R. D. (1977). The choice axiom after twenty years. Journal of Mathematical Psychology, 15, 215-233.

Luce, R. D., Raiffa, H. (1957). Games and decisions: Introduction and critical survey. New York: Wiley.

Mishra, S., Umesh, U. N., \& Stem, D. E. (1993). Antecedents of the attraction effect: An information-processing approach. Journal of Marketing Research, 30, 331-349.

Nash, J. F. (1950). The bargaining problem. Econometrica, 18, 155-162.

Rapoport, A., Guyer, M. J., \& Gordon, D. G. (1976). The $2 \times 2$ game. Ann Arbor, MI: University of Michigan Press.

Savage, L. J. (1972). The foundations of statistics (2nd ed.). New York: Dover. (Original work published 1954)

Schelling, T. C. (1960). The strategy of conflict. Cambridge, MA: Harvard University Press.

Sen, A. K. (1969). Quasi-transitivity, rational choice and collective decisions. Review of Economic Studies, 36, 381-393.

Sen, A. K. (1970). Collective choice and social welfare. San Francisco, CA: Holden-Day.

Sen, A. K. (2002). Internal consistency of choice. In A. K. Sen, Rationality and freedom (pp. 121-157). Cambridge, MA: Belknap Press.

Shafir, E., Simonson, I., \& Tversky, A. (1993). Reason-based choice. Cognition, 49, 11-36.

Shafir, E., \& Tversky A. (1992). Thinking through uncertainty: Nonconsequential reasoning and choice. Cognitive Psychology, 24, 449-474.

Sugden, R. (1985). Why be consistent? A critical analysis of consistency requirements in choice theory. Economica, 52, 167-183.

Sugden, R. (1993). Thinking as a team: Towards an explanation of nonselfish behavior. Social Philosophy and Policy, 10, 69-89. 
Sugden, R., \& Zamarrón, I. E. (2006). Finding the key: The riddle of focal points. Journal of Economic Psychology, 27, 609-621.

Tversky, A. (1972). Elimination by aspects: A theory of choice. Psychological Review, 79, 281299.

Tversky, A., \& Sattah, S. (1979). Preference trees. Psychological Review, 86, 542-573.

Tversky, A., \& Shafir, E. (1992). The disjunction effect in choice under uncertainty.

Psychological Science, 3, 305-309.

Tversky, A., \& Simonson, I. (1993). Context dependent preferences: The relative advantage model. Management Science, 39, 1179-1189.

Author Note

Briony D. Pulford, School of Psychology, University of Leicester; and Andrew M. Colman, School of Psychology, University of Leicester; Fergus Bolger, Durham Business School, University of Durham.

This project was supported by Grant RES-000-23-0154 from the Economic and Social Research Council of the UK. The authors would like to thank Clare Davies for help with data collection, Eldar Shafir, Robert Sugden, Ali al-Nowaihi, Sanjit Dhami, Karl Teigen, and other participants who provided useful comments on this research at the ESRC symposium on Confidence in Interactive Decisions, 1 July 2005, and anonymous referees for suggesting significant further improvements.

Correspondence concerning this article should be addressed to Andrew M. Colman, School of Psychology, University of Leicester, University Road, Leicester, LE1 7RH, United Kingdom. E-mail: amc@,le.ac.uk

\section{Footnotes}

1. The term dominance has subtly different though related denotations in decision theory and game theory. In this article, we are concerned only with strict dominance. In individual decision making, an Alternative $x$ (strictly) dominates another $y$ for a decision maker for whom $x$ is better than $y$ on all relevant attribute dimensions. In game theory, a Strategy $x$ (strictly) dominates another $y$ for a player who receives a better payoff from choosing $x$ than $y$ irrespective of the strategy or strategies chosen by the other player(s).

2. We originally included 12 games in this experiment, but in six of them large majorities of players preferred the $C$ strategies in the control condition, creating a ceiling effect and leaving little room for any increase in preferences for $C$ strategies when they became asymmetrically dominant in the other treatment conditions. An anonymous referee recommended relegating these games to a footnote. For the record, when all 12 games were included in the analysis, the differences between these three means were in the predicted direction but were not significant, $F(2,69)=2.06, p=.14$, effect size partial $\eta^{2}=.06$; but planned comparisons showed that the difference between the dominated-available and control conditions was significant, $t(46)=2.07$, $p<.05$ (two-tailed), and that the difference between the phantom decoy and control conditions was nonsignificant, $t(46)=1.06, p=.29$ (two-tailed).

3. When analyzing confidence ratings the confidence in $C$ choices were compared with the confidence in not- $C$ choices, thus incorporating the few $E$ choices made by participants in the dominated-available condition in with the $D$ choices.

4. An anonymous referee has suggested that this involved deception, but we respectfully disagree. Each Player I was indeed paired with another participant in the role of Player II, and the fact that this Player II was the same for every Player I did not affect the dyadic task that each 
player faced. Player II was also paired with another (randomly chosen) participant to calculate her payoffs. 


\section{Appendix A: Mathematical Derivation and Proofs}

Let $x$ Ry represent a binary relation denoting weak preference for $x$ over $y$, interpreted as " $x$ is at least as good as $y$ " or, what amounts to the same thing, " $y$ is not preferred to $x$ ". To specify an instance in which this weak preference relation does not hold, we write $\neg(x R y)$. Relations of strict preference $P$ and indifference $I$ are defined in terms of the weak preference relation as follows:

$$
\begin{gathered}
\text { Strict preference: }[x P y \Leftrightarrow[x R y \wedge \neg(y R x)]] ; \\
\text { Indifference: }[x I y \Leftrightarrow[x R y \wedge y R x]] .
\end{gathered}
$$

If $X$ is an arbitrary set, and $X \times X$ is the Cartesian product of ordered pairs $(x, y), x, y \in X$, then a binary relation $R$ on $X$ is simply a subset $R \subseteq\{X \times X\}$. Given a binary relation defined in this way, $(x, y) \in R$ iff the ordered pair belongs to the relation $R$, in which case we write $x R y$.

In accordance with the first postulate P1 of Savage's subjective expected utility (SEU) theory (Savage, 1954/1972, p. 18), a preference relation $R$ must satisfy the following minimal pair of conditions:

$$
\text { Connectedness: }[x R y \vee y R x], \forall x, y \in X \text {; }
$$

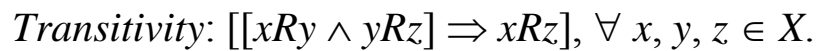

These two conditions ensure that the relation $R$ is what Savage called a simple ordering on $X$. The relation $R$ also satisfies a condition called reflexivity, because any alternative is trivially at least as good as itself:

\section{Reflexivity: $x R x \forall x \in X$.}

The derived preference relations $P$ and $I$ are both transitive, and $I$ is reflexive, but neither $P$ nor $I$ is connected. We now define a choice function $c(X)$ that assigns a choice set $C(Y, R)$ to every non-empty feasible subset $Y$ of $X$, such that

$$
[x \in C(Y, R) \Leftrightarrow x R y] \forall y \in Y .
$$

We interpret $Y$ as the feasible subset of the universal set $X$, to model choice situations in which some potential alternatives may be unavailable for choice. For example, in a holiday brochure, some holidays may be unavailable at certain times of the year, or in a supermarket or restaurant, some items may be out of stock or unavailable. If there is only one feasible alternative that is weakly preferred to all feasible alternatives, then the choice set $C(Y, R)$ is a singleton. In general, if $C(Y, R)$ contains two or more elements, then any member of the choice set may be chosen.

If $R$ is connected, transitive, and reflexive, and if $X$ is finite, then $C(Y, R)$ is non-empty and the choice function $c(X)$ is therefore defined over $X$. The following inductive proof is due to Sen (1969, Theorem II). Let the elements of $Y \subseteq X$ be $x_{1}, \ldots, x_{n}$. Consider first the pair $\left(x_{1}, x_{2}\right)$. By reflexivity and connectedness of $R$, either $\left[x_{1} R x_{1} \wedge x_{1} R x_{2}\right]$, in which case $x_{1} \in C(Y, R)$, or $\left[x_{2} R x_{1}\right.$ $\left.\wedge x_{2} R x_{2}\right]$, in which case $x_{2} \in C(Y, R)$. In either case, $C(Y, R)$ is non-empty. We now show that if $C(Y, R)$ is non-empty for $Y=\left(x_{1}, \ldots, x_{j}\right)$, then it is non-empty for $Y=\left(x_{1}, \ldots, x_{j}, x_{j+1}\right)$. Let $a_{j} R x_{k}$ for $k=1, \ldots, j$. Either $a_{j} R x_{j+1}$ or $x_{j+1} P a_{j}$. If $a_{j} R x_{j+1}$, then $a_{j} R x_{k}$ for $k=1, \ldots, j+1$, and $C(Y, R)$ is nonempty for $Y=\left(x_{1}, \ldots, x_{j}, x_{j+1}\right)$. If $x_{j+1} P a_{j}$, then $x_{j+1} R x_{k}$ for $k=1, \ldots, j+1$ unless $x_{k} P x_{j+1}$ for some $k=$ $1, \ldots j$, and by transitivity of $P, x_{k} P a_{j}$, which contradicts the assumption that $a_{j} R x_{k}$ for $k=1, \ldots, j$. Thus, once again, $C(Y, R)$ is non-empty for $Y=\left(x_{1}, \ldots, x_{j}, x_{j+1}\right)$, and this completes the proof.

At a formal level, the asymmetric dominance effect is defined by the following pair of choices by a decision maker:

$$
\begin{gathered}
C(\{x, y\})=\{x\}, \\
C(\{x, y, z\})=\{y\} .
\end{gathered}
$$

We are now ready to specify the basic contraction property of rational choice that is violated by this effect. It was introduced by Sen (1969) as Property $\alpha$.

$$
\text { Property } \alpha:[[x \in C(X, R) \wedge x \in Y \subseteq X] \Rightarrow x \in C(Y, R)] \text {. }
$$


Property $\alpha$ asserts that if some element is in the choice set of a particular set $X$, then it must also be in the choice set of a subset $Y$ of $X$. We are now ready to prove that Property $\alpha$ is implied by Savage's (1954/1972) first postulate, which formalizes the most minimal axiom of rational choice.

Consider a binary relation $R$ satisfying the conditions of a simple ordering on $X$ and a subset $Y \subseteq X$, with $x, y \in Y$. Suppose $x \in C(X, R)$ and $y \in C(Y, R)$. Then $[[x \in C(X, R) \wedge y \in X] \Rightarrow x R y]$, and $[[y \in C(Y, R) \wedge x \in Y] \Rightarrow y R x]$. By definition of $I,[[x R y \wedge y R x] \Rightarrow x I y]$, and by transitivity of $I$ and $R,[[x I y \wedge y R z] \Rightarrow x R z] \forall z \in Y$, therefore $x \in C(Y, R)$, as required by Property $\alpha$. This completes the proof. 
Appendix B: Games Used in Experiments 1 and 2

II

II

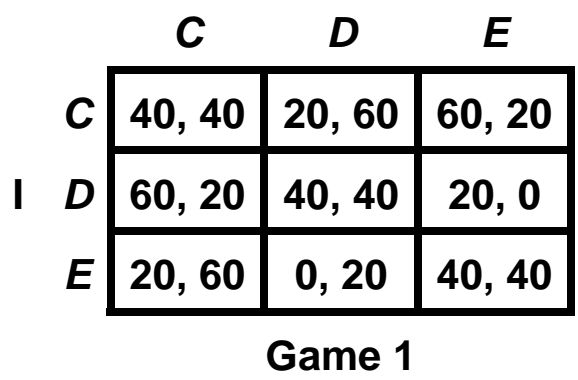

II

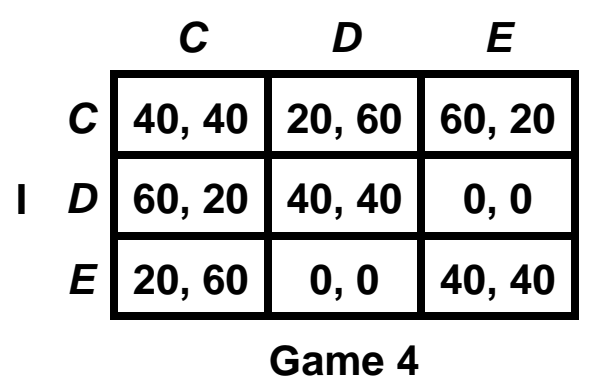

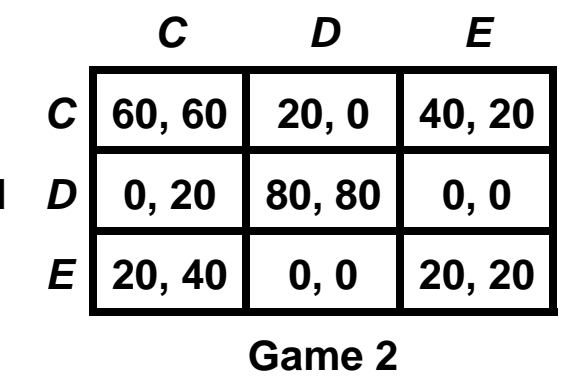

II

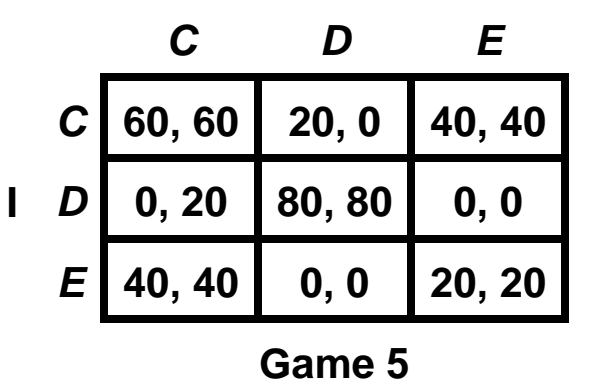

II

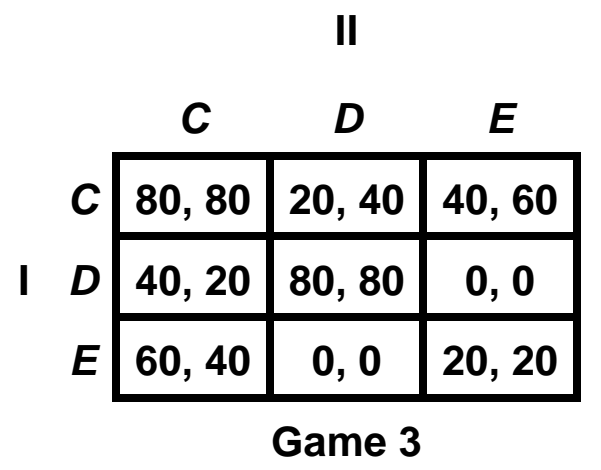

II

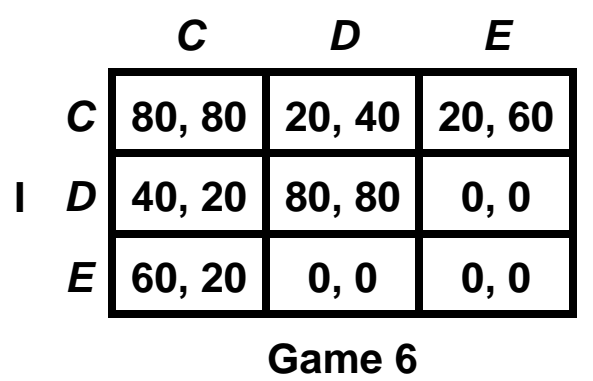


Games Used in Experiment 2

$$
\text { II }
$$

II

II

II

\begin{tabular}{|c|c|c|c|}
\hline & $C$ & $D$ & $E$ \\
\hline C & 80,60 & 20,40 & 60,40 \\
\hline I D & 40,20 & 60,80 & 0,0 \\
\hline$E$ & 60,60 & 0,0 & 40,40 \\
\hline
\end{tabular}

\begin{tabular}{|c|c|c|c|}
\hline & $C$ & $D$ & $E$ \\
\hline$C$ & 80,60 & 20,40 & 60,40 \\
\hline$D$ & 60,20 & 40,80 & 0,0 \\
\hline$E$ & 40,60 & 0,0 & 40,40 \\
\hline
\end{tabular}

\begin{tabular}{|c|c|c|c|}
\hline & $C$ & $D$ & $E$ \\
\hline C & 40,60 & 60,80 & 60,40 \\
\hline I $D$ & 80,40 & 20,20 & 0,0 \\
\hline$E$ & 20,60 & 40,0 & 40,40 \\
\hline
\end{tabular}

\begin{tabular}{|c|c|c|c|}
\hline & $C$ & $D$ & $E$ \\
\hline C & 60,80 & 40,20 & 60,60 \\
\hline I D & 80,40 & 20,60 & 0,0 \\
\hline$E$ & 20,60 & 20,0 & 40,40 \\
\hline
\end{tabular}

II

II

II

II

\begin{tabular}{|c|c|c|c|}
\hline & $C$ & $D$ & $E$ \\
\hline C & 60,60 & 40,20 & 60,40 \\
\hline I $D$ & 80,40 & 20,80 & 0,20 \\
\hline$E$ & 20,60 & 20,0 & 40,40 \\
\hline
\end{tabular}

\begin{tabular}{|c|c|c|c|}
\hline & $C$ & $D$ & $E$ \\
\hline C & 60,40 & 40,20 & 60,20 \\
\hline I D & 80,60 & 20,80 & 0,40 \\
\hline$E$ & 40,60 & 20,0 & 40,40 \\
\hline
\end{tabular}

\begin{tabular}{|c|c|c|c|}
\hline & $C$ & $D$ & $E$ \\
\hline$C$ & 40,80 & 80,20 & 60,40 \\
\hline$D$ & 60,40 & 20,60 & 0,20 \\
\hline$E$ & 20,60 & 40,0 & 40,40 \\
\hline
\end{tabular}

\begin{tabular}{|c|c|c|c|}
\hline & $C$ & $D$ & $E$ \\
\hline$C$ & 40,80 & 60,20 & 60,40 \\
\hline I D & 80,40 & 20,60 & 0,20 \\
\hline$E$ & 20,60 & 40,0 & 40,40 \\
\hline
\end{tabular}


II

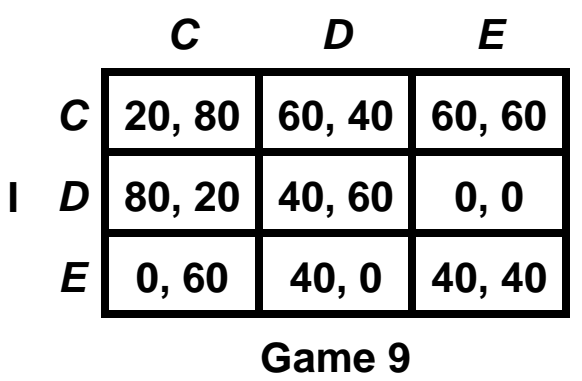

II

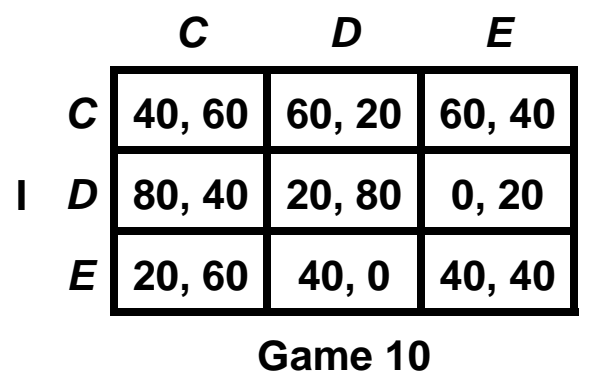

II

\begin{tabular}{|c|c|c|c|}
\hline & $C$ & $D$ & $E$ \\
\hline$C$ & 20,80 & 60,60 & 60,60 \\
\hline$D$ & 80,20 & 40,40 & 0,0 \\
\hline$E$ & 0,60 & 40,0 & 40,40 \\
\hline
\end{tabular}

II

\begin{tabular}{|c|c|c|c|}
\hline & $C$ & $D$ & $E$ \\
\hline$C$ & 40,40 & 60,20 & 60,20 \\
\hline$D$ & 80,60 & 20,80 & 0,20 \\
\hline$E$ & 20,60 & 40,0 & 40,40 \\
\hline
\end{tabular}

Note. All games are shown with asymmetrically dominant strategies in row and column $C$ and dominated strategies in row and column $E$. In the experiments, rows and columns were rotated to control for strategy order effects. In the control condition, E strategies were deleted. Payoffs represent pence sterling. 